\title{
Cortical folding influences migraine aura symptoms in CADASIL
}

\author{
Eric Jouvent, ${ }^{1,2,3}$ Jean-François Mangin, ${ }^{3}$ Dominique Hervé,, ${ }^{1,2}$ Marco Düring, ${ }^{4}$ \\ Martin Dichgans, ${ }^{4}$ Hugues Chabriat ${ }^{1,2}$
}

${ }^{1}$ Univ Paris Diderot, Sorbonne Paris Cité, UMR 740 INSERM, France

${ }^{2}$ Department of Neurology, AP-HP, Lariboisière Hospital, Paris, France

${ }^{3}$ LNAO, Neurospin, I2BM, CEA, France

${ }^{4}$ Institute for Stroke and Dementia Research, Medical Centre,

Ludwig-Maximilians-University, Munich, Germany

\section{Correspondence to}

Professor H Chabriat, Service de Neurologie, Hôpital Lariboisière, 2 rue Ambroise Paré, 75010

Paris, France;

hugues.chabriat@lrb.aphp.fr

Received 24 June 2011

Revised 14 September 2011

Accepted 2 October 2011

Published Online First

9 November 2011

\begin{abstract}
Objective Migraine with aura is a hallmark of cerebral autosomal dominant arteriopathy with subcortical infarcts and leukoencephalopathy (CADASIL). In contrast with the majority of CADASIL patients, some affected subjects never experience visual symptoms during their attacks of migraine with aura. The aim of this study was to determine whether specific morphology of the primary visual cortex is associated with the absence of visual symptoms during migraine aura in CADASIL.
\end{abstract}

Methods Patients from a large cohort of CADASIL patients, aged $<45$ years, and with a modified Rankin's scale $\leq 1$ were included in the study. Width and depth of the calcarine sulcus in the primary visual cortex as well as cortical thickness in its neighbourhood were compared between patients with visual and those with non-visual migraine auras.

Results 31 patients had visual symptoms (VA group) while nine reported only non-visual symptoms (NVA group) during their migraine auras. Asymmetry index of the calcarine sulcal depth largely differed between the NVA group and the VA group $(0.22 \pm 0.1 \mathrm{vs}-0.004 \pm 0.2$; $\left.p=1.7 \times 10^{-6}\right)$. The width of the right calcarine sulcus was significantly lower in the VA group $(p=0.04)$ and cortical thickness was larger in the NVA group $(p=0.03)$. Conclusion The absence of visual symptoms during migraine auras was associated with a profound asymmetry of the primary visual cortex. Aura symptoms seem to be linked to the morphology of the primary visual cortex in CADASIL. This finding potentially reflects more general relationships between spreading depression and cortex morphology in migraine with aura.

\section{INTRODUCTION}

There is convincing evidence that aura symptoms reported during attacks of migraine with aura (MA) are related to cortical spreading depression (CSD). ${ }^{1}$ Most often, CSD starts in the occipital cortex leading to visual symptoms at the onset of aura before the occurrence of sensory or aphasic symptoms. ${ }^{2}$ In experimental models, cortical folding was found to influence the extent of spreading depression over the cortex. ${ }^{3}$ Some authors have proposed a physical model to explain the usual sparing of the motor cortex based on the shape of Rolando's sulcus. ${ }^{4}$ Cerebral autosomal dominant arteriopathy with subcortical infarcts and leukoencephalopathy (CADASIL) is a rare hereditary small vessel disease of the brain. ${ }^{5} \mathrm{MA}$ is a major and early symptom of this disorder and is reported five times more frequently by affected subjects than in the general population. ${ }^{6}$ Although visual aura is the most frequent aura symptom in CADASIL patients, some affected individuals do not experience any visual symptoms during their attacks of MA. We hypothesised that morphological features of the calcarine sulcus might be associated with the occurrence or absence of visual symptoms during attacks of MA in CADASIL patients.

\section{METHODS}

\section{Subjects}

Subjects were drawn from an ongoing prospective study in two different centres, in Paris, France and Munich, Germany, the details of which have been reported previously. ${ }^{7}$ All patients were interviewed in both centres by a neurologist experienced in questioning patients suffering from headache using a structured interview. The diagnostic criteria of the International Headache Society (ICHD-2) were used to define the main characteristics of attacks of migraine. $^{8}$ The following data were specifically collected for aura symptoms in each individual: age at first aura, frequency of aura, duration of aura symptoms, presence or absence of gradual onset for each symptom, and presence or absence of visual, sensory, motor or aphasic manifestations. Only patients with MA according to ICHD-2 criteria were included in the present study. To avoid any confounding effect related to age or disease severity, the inclusion was restricted to patients with age $<45$ years and without disability (modified Rankin's Scale $\leq 1)$. Clinical status was assessed using the Mini-Mental Score Examination, Mattis Dementia Rating Scale and time to complete Trail Making Test version $\mathrm{B}$, as previously reported. ${ }^{7}$ An independent ethics committee approved this study in each centre.

\section{Data acquisition and processing}

The imaging protocol has been detailed elsewhere. ${ }^{9}$ The reconstruction of cortical sulci was made using BrainVisa software (http://brainvisa.info) with isotropic millimetric three-dimensional $T_{1}$ scans that allows the reconstruction and identification of 57 different sulci on each hemisphere. ${ }^{10}$ The results were then carefully checked on both two-dimensional segmentation masks and three-dimensional reconstruction of sulcal shapes in each subject. ${ }^{11}$ Specific attention was given to the reconstruction of the calcarine sulcus on both sides. Depth and width were extracted for all sulci on both sides as well as cortical thickness measured in the neighbourhood of the different sulci skeletons. ${ }^{11}$ The main steps of the methodology are illustrated in 


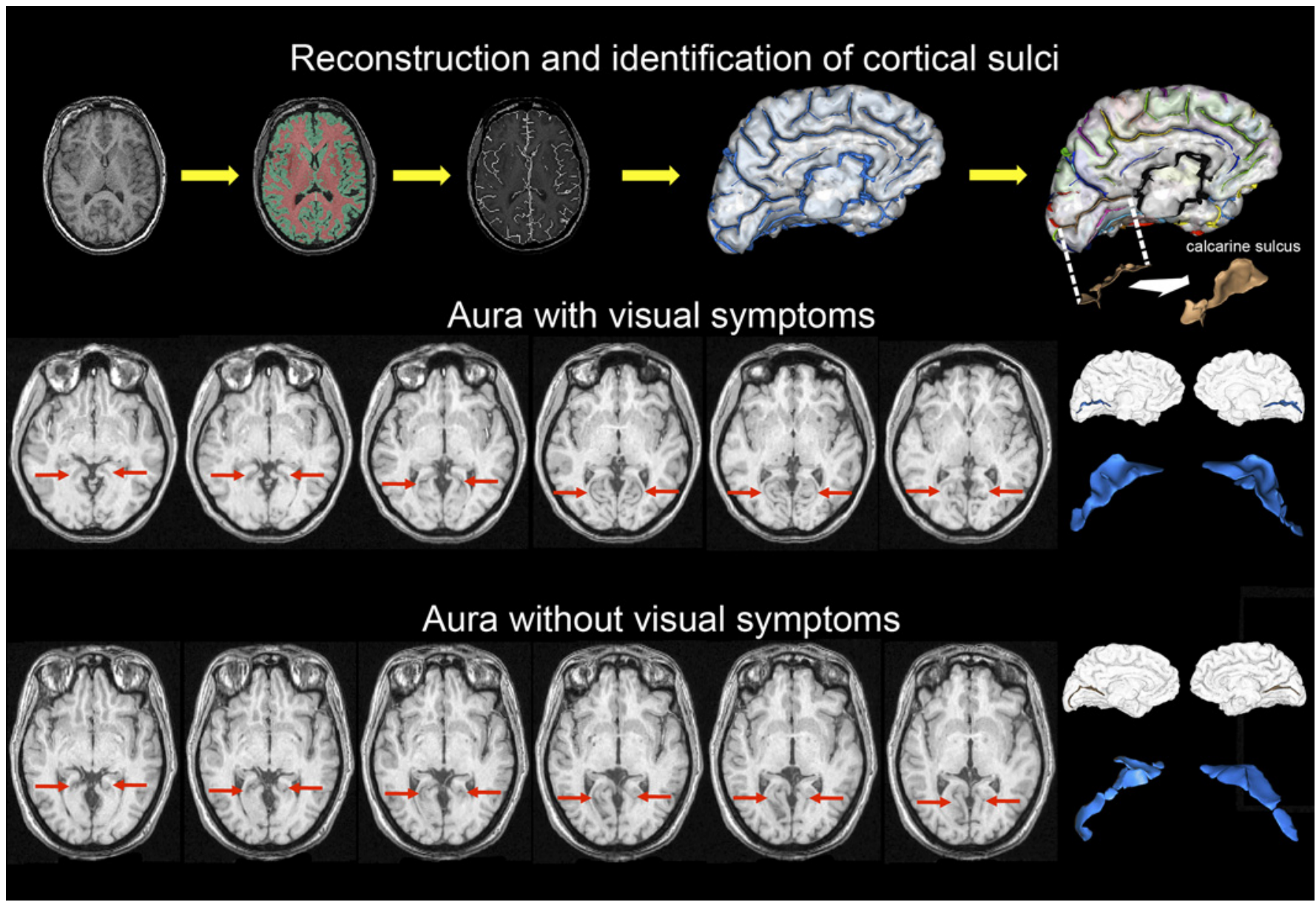

Figure 1 Sulcal reconstruction and identification. Principles and application for two randomly chosen patients (with or without visual symptoms during auras). After segmentation of brain voxels, grey and white matter voxels are determined and sulci skeletons reconstructed (top). Afterwards, sulci are identified. For the two patients, the calcarine sulcus is outlined with red arrows on native brain MRI. Three-dimensional reconstruction of the calcarine sulcus is shown for both sides. Note the difference in shape between the left and right calcarine sulci in the patient without visual symptoms during auras compared with the symmetry of both sides in the patient with visual symptoms during attacks.

figure 1. Masks of white matter T2 hyperintensities and of lacunar infarctions as well as the number of microhaemorrhages were determined using a semi-automated methodology. ${ }^{12}$ All lesion masks were subsequently validated by experienced readers. Volume of white matter hyperintensities, that of lacunar lesions and number of microhaemorrhages were recorded. Finally, the brain parenchymal fraction was calculated as the ratio of brain volume over that of intracranial cavity volume.

\section{Statistical methods}

All statistical analyses were made using $\mathrm{R}$ (http://cran.org). Group comparisons were made according to the variable type and distribution. For numerical variables, t tests were used when variables were normally distributed, taking into account the equal or unequal variances of the two groups. When assumption of normality was violated, the Wilcoxon rank sum tests were used. For categorical variables, $\chi^{2}$ tests were used.

\section{RESULTS}

Patient characteristics

Forty-two patients whose mean age was 36 years fulfilled the inclusion criteria. Two patients were excluded because of incomplete dataset $(n=1)$ or insufficient quality of images $(n=1)$. Among the remaining 40 subjects, 31 reported attacks of $\mathrm{MA}$, including visual symptoms (VA group) and nine attacks of
MA but without visual symptoms (NVA group). Patient characteristics are given in table 1. Age, gender, main characteristics of aura symptoms, disease severity and MRI markers did not significantly differ between the two groups (see table 1).

\section{Morphology of the calcarine sulcus}

The depth of the left calcarine sulcus was found to be significantly smaller in the NVA group than in the VA group (14.77 \pm 1.5 vs $17.18 \pm 2.7 ; p=0.002)$. In contrast, that of the right calcarine sulcus appeared larger in the NVA group than in the VA group although the difference did not reach statistical significance (17.94 \pm 1.0 vs $16.88 \pm 2.7 ; p=0.08$ ) (figure 1 ). The asymmetry index of sulcal depth, defined as (right depth-left depth)/right depth, was found to be close to 0 in the VA group but much higher in the NVA group $(-0.004 \pm 0.2$ vs $0.22 \pm 0.1$; $\left.\mathrm{p}=1.7 \times 10^{-6}\right)$. The width of the right calcarine sulcus was also significantly lower in the VA group $(1.07 \pm 0.2$ vs $1.25 \pm 0.3$; $\mathrm{p}=0.04)$ and cortical thickness around the left calcarine sulcus was larger in the NVA group (2.31 \pm 0.1 vs $2.18 \pm 0.1 ; p=0.03$ ) The asymmetry index of the calcarine sulcal width and cortical thickness did not differ between the two groups (table 1).

To further evaluate whether these results could be found only by chance, we also tested whether a similar difference was detectable for depth, width or cortical thickness on any side for the 14 major primary and secondary cortical sulci (superior and inferior frontal, central, post central, intraparietal, occipital, 
Table 1 Characteristics of the two groups of patients

\begin{tabular}{|c|c|c|c|}
\hline & $\begin{array}{l}\text { Patient with aura without visual } \\
\text { symptoms }(n=9)\end{array}$ & $\begin{array}{l}\text { Patient with aura and visual } \\
\text { symptoms }(n=31)\end{array}$ & p Value \\
\hline \multicolumn{4}{|l|}{ Clinical variables } \\
\hline Age (years) (mean $\pm S D$, range) & $36.1 \pm 6.5(24.7-44.7)$ & $36.6 \pm 4.9(25.3-43.6)$ & 0.84 \\
\hline Male gender (n (\%)) & $3 / 9(33)$ & $5 / 31(16)$ & 0.51 \\
\hline mRS (mean, median, range) & $0.0,0,0-0$ & $0.19,0,0-1$ & 0.17 \\
\hline MDRS (mean, median, range) & $141.4,142,136-144$ & $142,142,134-144$ & 0.78 \\
\hline MMSE (mean, median, range) & $28.3,29,26-30$ & $29,29.5,25-30$ & 0.26 \\
\hline TMTB time to complete (mean, median, range) & $60.5,59.5,37-92$ & $72.29,60,24-271$ & 0.42 \\
\hline History of stroke $(\mathrm{n}(\%))$ & $3 / 9(33)$ & $11 / 31(36)$ & 0.78 \\
\hline History of TIA† (n (\%)) & $3 / 9(33)$ & $7 / 24(29)$ & 0.83 \\
\hline Lack of any cerebrovascular event (n (\%)) & $4 / 9(44)$ & $15 / 31(48)$ & 0.87 \\
\hline Systolic blood pressure (mean \pm SD) & $124.0 \pm 19.7$ & $122.3 \pm 13.4$ & 0.81 \\
\hline Diastolic blood pressure (mean $\pm \mathrm{SD}$ ) & $69.0 \pm 13.1$ & $71.5 \pm 11.0$ & 0.60 \\
\hline Hypercholesterolemia $\neq(\mathrm{n}(\%))$ & $2 / 9(22)$ & $7 / 31(23)$ & 0.67 \\
\hline Diabetes§ (n (\%)) & $1 / 9(11)$ & $0 / 31(0)$ & 0.51 \\
\hline Paris centre $(\mathrm{n}(\%))$ & $7 / 9(78)$ & $17 / 31(55)$ & 0.40 \\
\hline \multicolumn{4}{|l|}{ Type of auras (n (\%)) } \\
\hline Onset before 30 years & $5 / 9(55)$ & $23 / 31(75)$ & 0.51 \\
\hline Attack frequency $>1 /$ month & $1 / 9(11)$ & $8 / 31(25)$ & 0.52 \\
\hline Gradual onset & $8 / 9(88)$ & $27 / 31(87)$ & 0.91 \\
\hline Sensory symptoms & $9 / 9(100)$ & $23 / 31(75)$ & 0.22 \\
\hline Aphasic symptoms & $6 / 9(67)$ & $20 / 31(65)$ & 0.78 \\
\hline Motor symptoms & $5 / 9(55)$ & $6 / 31(19)$ & 0.09 \\
\hline \multicolumn{4}{|l|}{ MRI markers } \\
\hline Volume of $\mathrm{LL}\left(\mathrm{mm}^{3}\right)$ (mean, median, range) & $180.8,14.0,0-1194.0$ & $275.6,2.1,0-2307.2$ & 0.77 \\
\hline Volume of WMH $\left(\mathrm{mm}^{3}\right)$ (mean, median, range) & $42362.1,37411.8,7057.5-74590.1$ & $48352.7,46753.6,5183.3-151160.7$ & 0.87 \\
\hline Presence of $\mathrm{CM}$ & & & 0.24 \\
\hline No & $7 / 9(78)$ & $30 / 31(97)$ & \\
\hline Yes & 2/9 (22) & $1 / 31(3)$ & \\
\hline $\mathrm{BPF}(\mathrm{mean} \pm \mathrm{SD})$ & $89.8 \pm 4.2$ & $89.1 \pm 3.8$ & 0.66 \\
\hline \multicolumn{4}{|l|}{ Calcarine sulcus morphology } \\
\hline \multicolumn{4}{|l|}{ Depth $($ mean $\pm S D)$} \\
\hline Right & $17.94 \pm 1.0$ & $16.88 \pm 2.7$ & 0.08 \\
\hline Left & $14.77 \pm 1.5$ & $17.18 \pm 2.7$ & 0.002 \\
\hline Asymmetry index & & & $1.7 \times 10^{-6}$ \\
\hline \multicolumn{4}{|l|}{ Width $($ mean $\pm S D)$} \\
\hline Right & $1.07 \pm 0.2$ & $1.25 \pm 0.3$ & 0.04 \\
\hline Left & $1.18 \pm 0.2$ & $1.30 \pm 0.4$ & 0.23 \\
\hline Asymmetry index & & & 0.27 \\
\hline \multicolumn{4}{|l|}{ Cortical thickness (mean $\pm S D$ ) } \\
\hline Right & $2.36 \pm 0.1$ & $2.26 \pm 0.2$ & 0.11 \\
\hline Left & $2.31 \pm 0.1$ & $2.18 \pm 0.1$ & 0.03 \\
\hline Asymmetry index & & & 0.46 \\
\hline
\end{tabular}

*Focal neurological deficit with acute onset, lasting at least $24 \mathrm{~h}$, confirmed by imaging (CT scan or MRI).

†Focal neurological deficit with acute onset, lasting less than $24 \mathrm{~h}$, without visible lesion on MRI.

$\ddagger$ Hypercholesterolaemia confirmed by biological evaluation or taking lipid lowering drugs.

$\S 1997$ WHO criteria.

BPF, brain parenchymal fraction; CM, cerebral microhaemorrhages; LL, lacunar lesions; MDRS, Mattis Dementia Rating Scale; MMSE, Mini-Mental Score Examination; mRS, modified Rankin's Scale; TIA, transient ischaemic attack; TMTB, Trail Making Test version B; WMH, white matter hyperintensities.

sylvian, superior and inferior temporal, cingulate, parietooccipital, collateral, orbitofrontal, olfactory). ${ }^{13}$ No significant difference was observed for any parameter or location between the two groups except for the width of the right parietooccipital sulcus $(p=0.02)$ which corresponds to a single test with $p<0.05$ over 84 tests.

\section{DISCUSSION}

In the present study, we found that the primary visual cortex of patients who do not experience visual symptoms during migraine aura is strikingly asymmetrical compared with that of the VA group. CADASIL has been shown to promote the occurrence of CSD in Notch3 mutated mice ${ }^{14}$ but the present results suggest that some specific morphological features of calcarine sulci and occipital cortex on both sides may prevent the occurrence of CSD in CADASIL patients. This is in line with some experimental data showing that CSD does not spread towards the motor frontal areas due to the shape of the central sulcus. ${ }^{4}$ However, whether these morphological features actually determine the occurrence of visual symptoms during auras or 
just reflect other underlying mechanisms at the cortical level warrants further investigations.

The depth of the left calcarine sulcus was found to be $2.4 \mathrm{~mm}$ less in the NVA group compared with the other group. This difference, equivalent to a 30 year difference between the two groups, ${ }^{13}$ is unlikely to be related to cortical atrophy caused by the disease in young and non-disabled patients. These results may be related to some differences in brain development between patients. The occipital area is undergoing particular morphological changes during this period. In particular, the socalled Yakovlevian torque twists the left occipital pole across the midline with a larger growth than its right counterpart. ${ }^{15}$ Visual examination of the global shape of the calcarine sulcus showed important differences between the two groups which are unlikely to be related to the progression of the disease (see figure 1). It is unlikely that genotype explains our findings as types of mutations did not differ between the VA and NVA groups (data not shown).

This study has several strengths. The sample of patients was homogeneous. All post-processing techniques were used in the space of acquisition of data with visual checking at each stage of segmentation and reconstruction. ${ }^{11}$ This approach largely restrains the number of statistical tests and renders the interpretation of results more reliable. The methodology was also previously validated in the context of white matter lesions. ${ }^{11} 12$

This study also has several limitations. Firstly, the sample was of limited size. We chose to include only patients with minimal disability and age less than 45 years to reduce the heterogeneity in the extent of cerebral lesions that may unexpectedly affect the different post-processing methods. However, the results remained significant when choosing different cut-off values for age between 35 and 50 years or when the analyses were not constrained by the modified Rankin's Scale score. In addition, it is unlikely that the results were caused by chance as three of six tests were found to be statistically significant for the calcarine sulcus but only one of 84 tests for the 14 other major cortical sulci. Finally, in the NVA group there were only two patients from the Munich centre while 14 were included in the VA group. However, the results were found to be unchanged when the analysis was restricted to patients originating from Paris.

In summary, the results of this study suggest that the morphology of the primary visual cortex is associated with the occurrence of visual symptoms during MA in CADASIL. Further studies are needed to elucidate the exact link between the morphological aspects of the visual cortex and the occurrence of CSD in this disorder.

Funding This work was supported by PHRC grant AOR 02-001 (DRC/APHP) and performed with the help of ARNEVA (Association de Recherche en Neurologie VAsculaire), Hôpital Lariboisiere, France, 'La Fondation Planiol' and 'La Fondation NRJ-Institut de France'. EJ was supported by a grant from the Fonds d'Etude et de Recherche du Corps Médical-FERCM and the Société Française de Neurologie-SFN.

\section{Competing interests None.}

Ethics approval An independent ethics committee approved the study in each centre.

Contributors All authors contributed to data acquisition and processing. EJ and $\mathrm{HC}$ drafted the manuscript. All authors corrected the manuscript and approved the final version.

Provenance and peer review Not commissioned; externally peer reviewed.

\section{REFERENCES}

1. Schwedt TJ, Dodick DW. Advanced neuroimaging of migraine. Lancet Neurol 2009:8:560-8.

2. Russell MB, Olesen J. A nosographic analysis of the migraine aura in a general population. Brain 1996;119:355-61.

3. Bockhorst KH, Smith JM, Smith MI, et al. A quantitative analysis of cortical spreading depression events in the feline brain characterized with diffusion-weighted MRI. J Magn Reson Imaging 2000;12:722-33.

4. Grenier $\mathbf{E}$, Dronne MA, Descombes $\mathrm{S}$, et al. A numerical study of the blocking of migraine by Rolando sulcus. Prog Biophys Mol Biol 2008;97:54-9.

5. Chabriat H, Joutel A, Dichgans M, et al. Cadasil. Lancet Neurol 2009;8:643-53.

6. Vahedi K, Chabriat $\mathrm{H}$, Levy $\mathrm{C}$, et al. Migraine with aura and brain magnetic resonance imaging abnormalities in patients with CADASIL. Arch Neurol 2004:61:1237-40.

7. Viswanathan A, Godin 0, Jouvent E, et al. Impact of MRI markers in subcortical vascular dementia: a multi-modal analysis in CADASIL. Neurobiol Aging 2010;31:1629-36.

8. Headache Classification Subcommittee of the International Headache Society. The International Classification of Headache Disorders, 2nd Edn. Cephalalgia 2004:24(Suppl 1):9-160.

9. Epelbaum S, Benisty S, Reyes S, et al. Verbal memory impairment in subcortical ischemic vascular disease. A descriptive analysis in CADASIL. Neurobiol Aging 2011;32:2172-82.

10. Mangin JF, Jouvent E, Cachia A. In-vivo measurement of cortical morphology: means and meanings. Curr Opin Neurol 2010;23:359-67.

11. Jouvent $\mathbf{E}$, Reyes $\mathrm{S}$, Mangin JF, et al. Apathy is related to cortex morphology in CADASIL. A sulcal-based morphometry study. Neurology 2011;76:1472-7.

12. Jouvent $\mathbf{E}$, Mangin JF, Porcher R, et al. Cortical changes in cerebral small vessel diseases: a 3D MRI study of cortical morphology in CADASIL. Brain 2008; 131:2201-8.

13. Kochunov P, Mangin JF, Coyle T, et al. Age-related morphology trends of cortical sulci. Hum Brain Mapp 2005;26:210-20.

14. Eikermann-Haerter K, Yuzawa I, Dilekoz E, et al. Cerebral autosomal dominant arteriopathy with subcortical infarcts and leukoencephalopathy syndrome mutations increase susceptibility to spreading depression. Ann Neurol 2011;69:413-18.

15. Lyttelton OC, Karama S, Ad-Dab'bagh Y, et al. Positional and surface area asymmetry of the human cerebral cortex. Neuroimage 2009;46:895-903. 


\section{Cortical folding influences migraine aura} symptoms in CADASIL

Eric Jouvent, Jean-François Mangin, Dominique Hervé, Marco Düring, Martin Dichgans and Hugues Chabriat

J Neurol Neurosurg Psychiatry 2012 83: 213-216 originally published online November 9, 2011

doi: 10.1136/jnnp-2011-300825

Updated information and services can be found at:

http://jnnp.bmj.com/content/83/2/213

\section{These include:}

References This article cites 14 articles, 3 of which you can access for free at: http://jnnp.bmj.com/content/83/2/213\#BIBL

Email alerting Receive free email alerts when new articles cite this article. Sign up in the service box at the top right corner of the online article.

Topic Articles on similar topics can be found in the following collections Collections

\section{Notes}

To request permissions go to:

http://group.bmj.com/group/rights-licensing/permissions

To order reprints go to:

http://journals.bmj.com/cgi/reprintform

To subscribe to BMJ go to:

http://group.bmj.com/subscribe/ 